\title{
The "growing" evidence of the bottle and the bump: how does alcohol use during pregnancy affect infant and childhood growth?
}

\author{
Linda Marie O'Keeffe
}

Department of Epidemiology and Public Health, UCC

\section{A drink a day keeps the doctor away.....}

"Guinness is good for you!" And it's not just Guinness who, in the past, peddled this message as part of their global advertising campaign! In fact, pregnant women in Ireland were once advised to drink a glass of Guinness a day to fortify themselves and their babies! Although a practice that has been completely abandoned since the 1980s and one which would surely cause a stir today, it is astonishing to ever imagine pregnant women consuming alcohol on the recommendation of their doctor or midwife. Indeed, clinical trials of the potential use of alcohol to prevent preterm birth were on-going up until the 1970 s, and some health professionals used alcohol for this purpose in obstetric practice. These days there is a greater focus on the harmful effect of alcohol and media attention on the issue of "moderate" drinking during pregnancy has never been more intense. Today it is estimated that, in countries such as Ireland, the United Kingdom, Denmark and the Netherlands that of $50 \%$ of women continue to drink alcohol during pregnancy. But why do so many women continue to drink during pregnancy? Exactly how much are women drinking? Is it safe? And what level of consumption, if any, can women confidently consume without causing harm to their baby?

\section{He said. .. .she said. ... The problem of conflicting advice!}

There are a myriad of reasons why alcohol consumption remains common during pregnancy. While the majority of clinical and government guidelines suggest that pregnant women abstain from alcohol consumption during pregnancy, other guidelines such as National Institute of Health and Care Excellence [NICE] guidelines in the UK suggest that 1-2 drinks up to twice per week may not be harmful to the unborn baby. This contrasts with advice regarding other maternal behaviours during pregnancy such as smoking, about which there is no dispute in relation to its harmful effects on the unborn child. In addition, while many guidelines suggest that abstinence is the safest option for pregnant women, 
some evidence suggests that in practice not all healthcare professionals believe it is absolutely necessary to abstain from alcohol once levels of consumption are kept "moderate". However, the exact meaning of "moderate" drinking is highly subjective. Consequently, media or public health messages suggesting "moderate" amounts of alcohol could be safe in pregnancy must be carefully qualified with high quality research due to a significant risk of misinterpretation. This is important to ensure that women do not receive conflicting messages which can easily be mis-understood [see Figure 1].

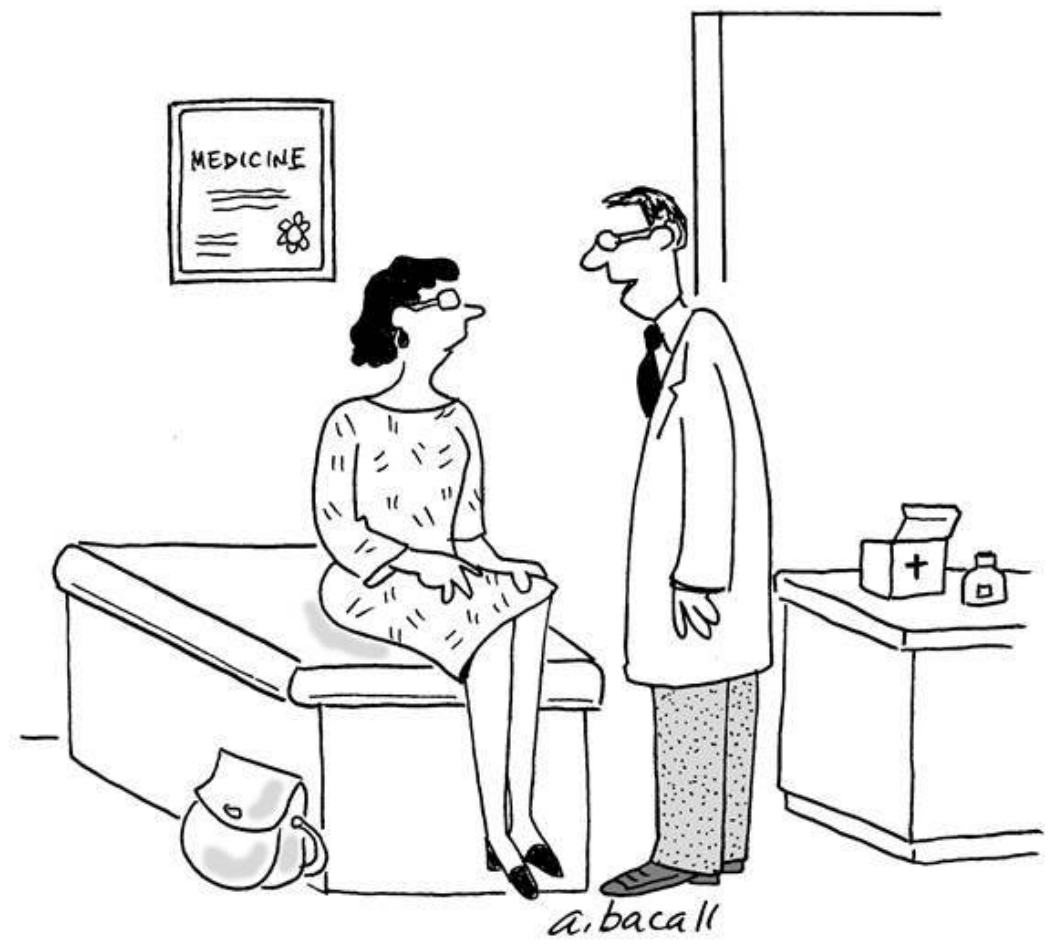

"Eat in moderation. Drink in moderation.

Be merry all you want."

Figure 1: Drink in moderation. Be merry all you want. Image: www.cartoonstock.com.

\section{Research that captures the truth}

The international lack of uniformity in clinical and government guidelines stems from a number of problems that arise at a research level. Firstly, scientists continue to find harmful, beneficial and equivocal effects of alcohol use during pregnancy which can all be independently explained by different biological evidence, thus making the effects of alcohol use during pregnancy difficult to disentangle. For example researchers who have found moderate alcohol use during pregnancy to be protective of preterm birth can substantiate this claim through biological evidence showing that alcohol may inhibit labour through release of hormones that relax the woman's uterus and prevent the baby being born too early. Alternatively, scientists who have found moderate drinking during pregnancy to 
increase the risk of preterm birth are able to link the increased risk among drinkers to the production of other hormones called prostaglandins which may mediate the association. Secondly, due to lack of a biological marker which tells us if women have been drinking during pregnancy, research relies on women's self-reported alcohol consumption which may often be underestimated or inaccurately reported. Women may underreport for many reasons, including a desire to answer in a socially acceptable way to avoid stigma or unintentionally under-reporting due to inability to recall consumption accurately. Responding to an interview about drinking during pregnancy in a socially acceptable way is more likely to occur if a woman is interviewed face to face, when responses can often be easily influenced by the way the questions are posed. Under-reporting is also likely in situations where alcohol data are collected after the woman has delivered her baby because alcohol consumption is not always habitual like other health behaviours such as smoking and therefore more likely to be recalled incorrectly. In addition, further problems can arise when a woman has had a negative infant health outcome and is interviewed after pregnancy on her behaviour during pregnancy. In particular, women with a negative experience may be more likely to scrutinize their behaviour during pregnancy and thus report differently to women with a positive infant health outcome. Consequently, it is sometimes difficult to separate what we believe to be a true association explained by some biological mechanism mediated, for example by hormones, or a spurious finding which is in fact a result of reporting biases as mentioned above.

A further problem that arises relates to a concept known as the "healthy drinker" phenomenon. Women who drink low amounts of alcohol during pregnancy may be healthier than women who stop consuming alcohol altogether when they recognize they are pregnant. This results in studies with conflicting results. For example, some studies have found that low to moderate drinking may be beneficial to the infant while others have found a harmful association with health outcomes. In studies where low levels of alcohol are shown to be beneficial, we cannot be sure that the "healthy drinker" effect is not at work. This means that healthier women may already be more likely to have a healthy baby because they are healthier in general and more socially advantaged (educated, older, married) rather than because they consume alcohol during pregnancy. This can often render the results of studies unreliable, inaccurate and even dangerous if misinterpreted by the media or women themselves.

\section{My research aims}

The challenges of collecting reliable and accurate data on alcohol use during pregnancy thus far mean that new approaches to measuring alcohol intake in pregnant women are required. In addition, we know very little about women's alcohol consumption patterns during pregnancy in Ireland. Finally, despite the proliferation of studies on the effects 
of alcohol use on infant health, we still do not fully understand the effects of alcohol on growth in infants and children.

As part of my PhD I will collect detailed information on alcohol use during pregnancy at Cork University Maternity Hospital (CUMH). I will also compare the use of alcohol reported in CUMH to other available Irish data in order to examine plausible prevalence estimates; this will include the use of the Screening for Pregnancy Endpoints Study (SCOPE) and the Growing up in Ireland (GUI) study. SCOPE is a large multi-centre follow-up study of 5,628 women recruited in Ireland, the United Kingdom, Australia and New Zealand. GUI is Ireland's first cohort study of almost 11,000 children.

In order to examine the potential impacts of low and moderate alcohol use during pregnancy on infant growth, I will use data from SCOPE, GUI and another dataset called the Avon Longitudinal Study of Parents and Children (ALSPAC). ALPSAC is a large British birth study of almost 15,000 women and children whom have been followed up from birth to adulthood. In SCOPE and GUI I will examine the effects of low and moderate alcohol use on growth outcomes at birth using a statistical technique called logistic regression. In ALSPAC I will examine the growth effects of alcohol use during pregnancy in children up to age 10 years using a statistical technique called multilevel modelling.

\section{Impact}

The first step in reducing alcohol prevalence during pregnancy and, subsequently, alcohol related harm in infants and children is to achieve coherence in guidelines on safe levels of alcohol during pregnancy by improving the quality of research conducted in this area. Furthermore, it is important that we as researchers communicate responsible and safe public health messages resulting from our work which clearly highlight flaws that may impact the reliability of findings. Taken together, these approaches offer substantial but realistic potential for impact both in Ireland and abroad in relation to maternal and child health.

I would like to thank my primary supervisors Professor Patricia Kearney and Professor Richard Greene as well as my other collaborators. I would also like to acknowledge the Health Research Board (HRB) and National Perinatal Epidemiology Centre (NPEC) for funding my PhD and supporting the collection of my data. Finally I would like to thank the staff of Cork University Maternity Hospital who facilitated my project and the participants of all studies used in my work. 